\title{
SECTION 8: OPTIONAL FURTHER STUDIES
}

\subsection{INTRODUCTION}

As part of the FIRI program, it was recognized that providing samples in sufficient quantity for laboratory procedures is close to ideal and does not represent "typical" conditions. Therefore, laboratories were asked to consider 2 optional studies: investigating the effects of sample size on results and achieving high precision. The sample size study was focused on Sample E, humic acid, which had been chosen because of the rigorous pretreatment it had undergone in the solution stage, and which would ensure sample homogeneity. The precision study was focused on Sample D, the Belfast dendro-dated wood sample, given its importance in the master calibration.

\subsubsection{Small Sample Size Results}

Laboratories were asked to provide results at the smallest sample size they would consider. This resulted in an additional 52 results from 27 laboratories. A summary of these is given in Table 8.1.

Table 8.1 Summary of small sample size age (yr BP) results for Sample E

\begin{tabular}{lllllll}
\hline $\mathrm{N}$ & Mean & Median & Minimum & Maximum & $\mathrm{Q} 1$ & $\mathrm{Q} 3$ \\
\hline 52 & 11,776 & 11,796 & 313 & 10,370 & 13,000 & 11,880 \\
\hline
\end{tabular}

A few laboratories provided more than 1 result. One (laboratory 15) provided 44 separate results for Sample E. These results are not included in the summary of the 52 results since this would bias the analysis. Laboratory 15 (AMS) provided results across a wide range of sample sizes, from their optimal size to the smallest size they would analyze.

\subsubsection{High-Precision Samples}

Laboratories were asked to provide high-precision results for the Belfast dendro-dated wood, Sample D. Two laboratories (15 and 25) indicated that their results were of high-precision - the ages of these were 4510 (10) BP and 4586 (28) BP, respectively. Given there were so few results identified as being reported with "high precision," no further analysis was completed on this part of the study.

\subsection{THE EFFECT OF SAMPLE SIZE}

In this section, the focus is on modelling the relationship between sample size and the absolute deviations from the consensus value for this sample. The effect of the quoted sigma on this relationship will also be explored. The AMS laboratories were best able to contribute to this study since they were able to report multiple results at a variety of sample sizes.

A total of 11 AMS laboratories gave 90 results, with almost half of these coming from a single laboratory (laboratory 15) and 6 laboratories giving 3 or fewer results. A scatterplot of the absolute deviations against the sample size (carbon mass) of the results is given in Figure 8.1. From the figure, we can see that results above $1 \mathrm{mg}$ tend to have much smaller deviations than results from smaller carbon mass samples.

Log transformation of both the sample sizes and the deviations were used to examine the relationship and to control the skew. A plot of the log transformed data is given in Figure 8.2. 


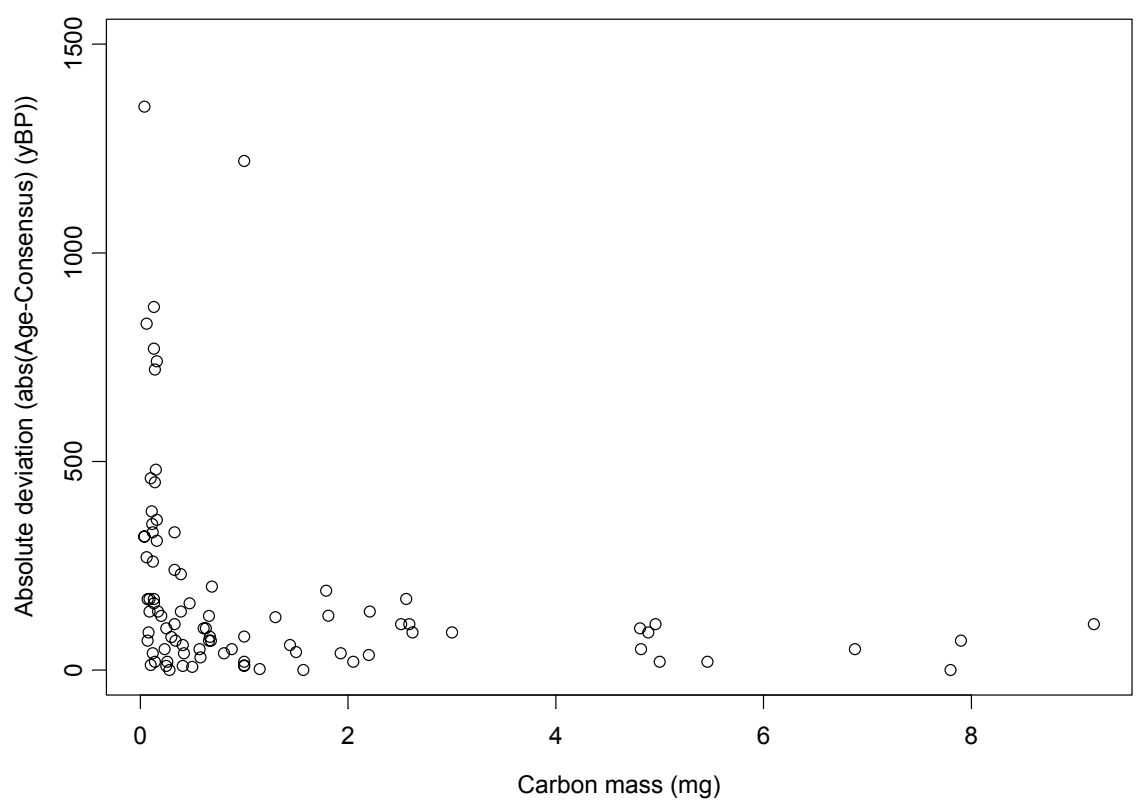

Figure 8.1 Scatterplot of sample size versus absolute deviation from consensus of Sample E results from AMS laboratories with at least 1 small sample size result

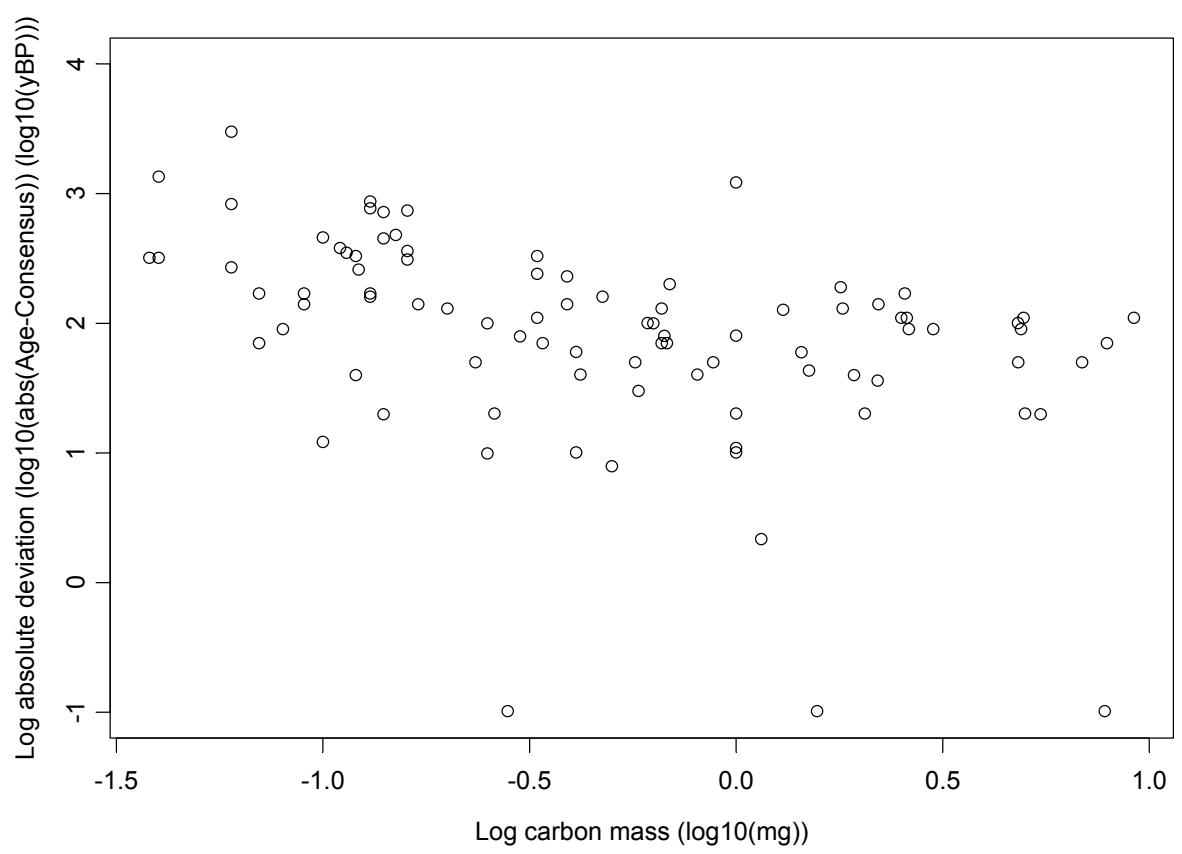

Figure 8.2 Scatterplot of $\log _{10}$ sample size versus $\log _{10}$ absolute deviation from consensus of Sample E results from AMS laboratories with at least 1 small sample size result

Figure 8.2 indicates that the log transformations of the data show a more linear relationship, which can then be modelled formally. A linear relationship was estimated and the resulting equation is:

$\log ($ absolute deviation $)=1.7559-0.4996 \times \log ($ carbon mass $)$ 
The $\mathrm{R}^{2}$ value is $16.4 \%$ (a measure of the variation in deviation explained by sample size), which is extremely low. Thus, sample size, although statistically significant, does not provide a good explanation of the scatter in the absolute deviation.

\subsubsection{Comments}

From this output, it can be seen that there is very strong evidence that the size of the sample does influence the average absolute standard deviation of the results via the $\log _{10}$ transformations of both the predictor and the response variable.

The analysis indicates that, although the regression is significant, it does not account for that much of the variation in the deviations. This is evident also from the low $\mathrm{R}^{2}$ value (only $16 \%$ ) for the model.

\subsection{THE EFFECT OF QUOTED ERROR AND SAMPLE SIZE}

So far, we have not considered the effect of the quoted sigma on this relationship. Two approaches to investigate this question are considered:

1. Scale the absolute deviations by the quoted sigma and re-analyze using the scaled values as the response;

2. Include quoted errors as another covariate in the regression modelling.

\subsubsection{Scaling the Deviation Using the Quoted Sigma}

Figure 8.4 indicates that by scaling the response by quoted sigma, there is much less of an evident pattern in the relationship with sample size; this is made more obvious when carbon mass is logged in Figure 8.5.

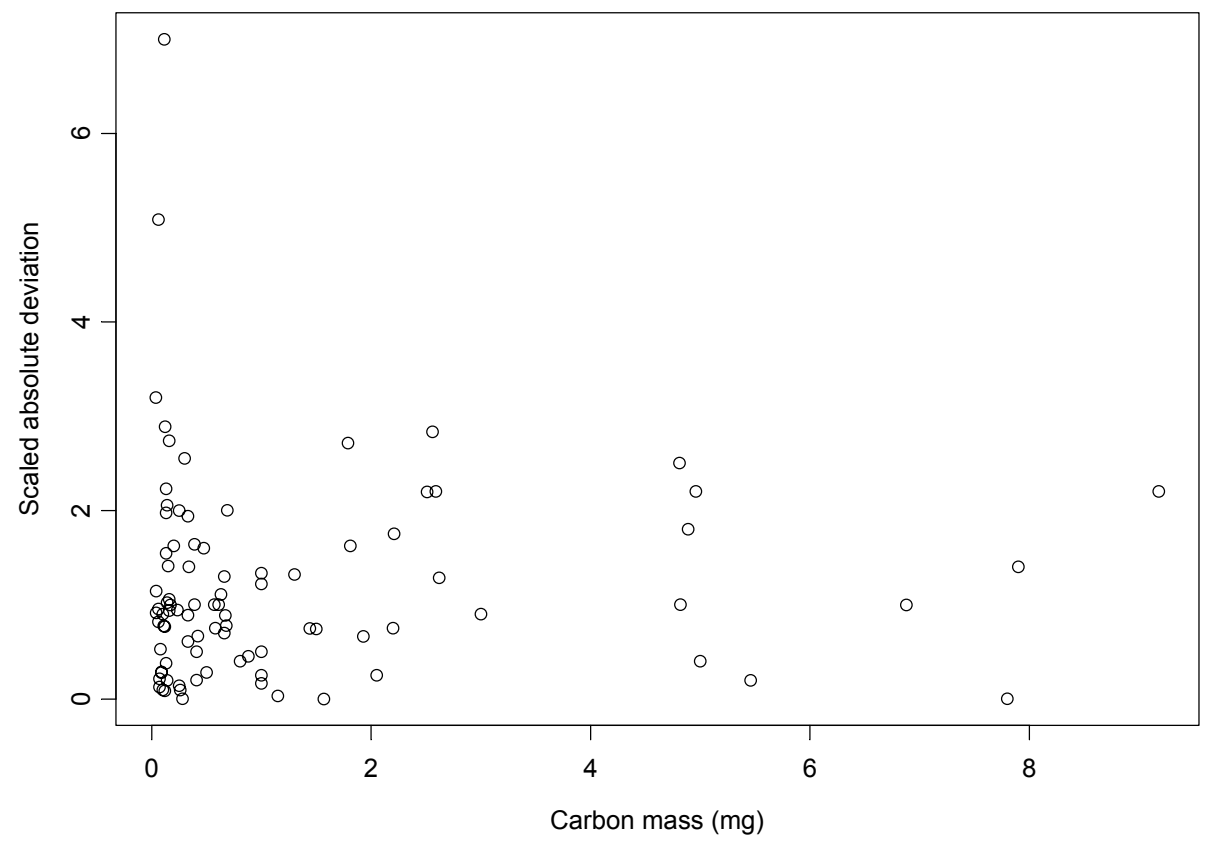

Figure 8.4 Scatterplot of scaled absolute deviation from the consensus versus the sample size of Sample E, results from AMS labs with at least 1 small sample size result 


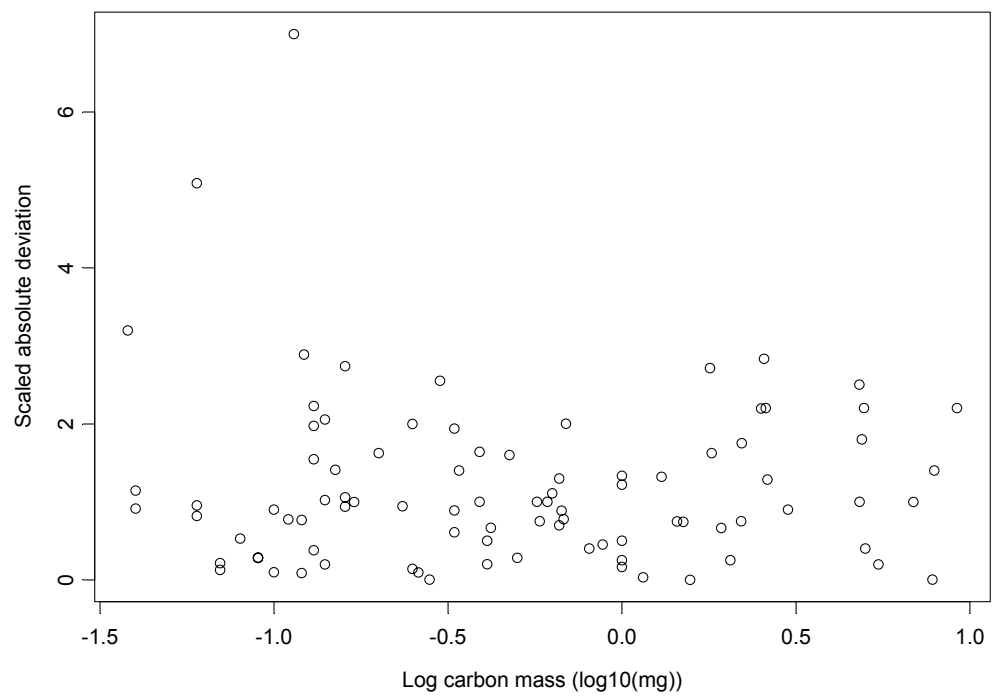

Figure 8.5 Scatterplot of scaled absolute deviation from the consensus versus the $\log _{10}$ sample size of Sample E, results from AMS labs with at least 1 small sample size result

This apparent lack of relationship is confirmed by the regression analysis, which gave a p-value for $\log$ (carbon mass) of 0.56 ; thus, we could conclude that there was no statistically significant relationship between the scaled deviation and the carbon mass. These results indicate that after scaling the absolute deviations by their associated quoted sigmas, there is no longer any dependence on sample size.

\subsubsection{Including Quoted Sigma as a Second Covariate in the Regression}

Figure 8.6 indicates that there does appear to be a linear relationship between the $\log _{10}$ quoted sigma and the $\log _{10}$ absolute deviation from the consensus. The model with the $\log _{10}$ quoted sigma added as a covariate showed that there was not a statistically significant relationship with the carbon mass $(\mathrm{p}=0.65)$, but that there was one with the quoted sigma $(\mathrm{p}<0.05)$.

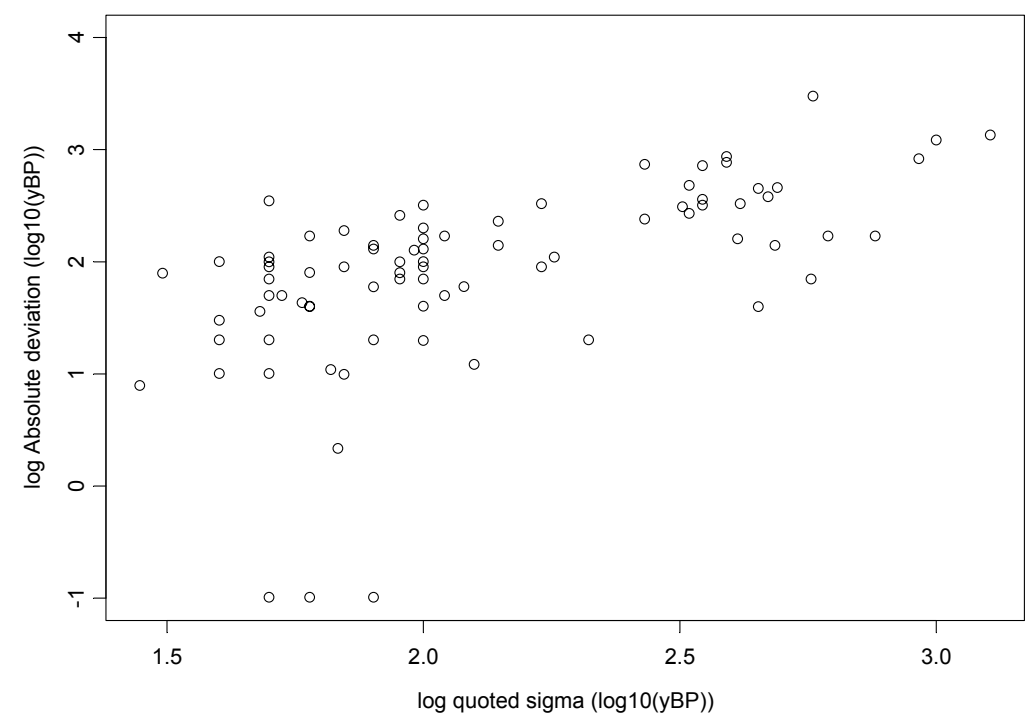

Figure 8.6 Scatterplot of $\log _{10}$ absolute deviation from consensus versus $\log _{10}$ quoted sigma of Sample E, results from AMS labs with at least 1 small sample size result 
The $\log _{10}$ sample size is no longer a significant predictor, the but $\log _{10}$ quoted sigma is highly significant, indicating that the quoted sigma is better than sample size as a predictor, and that both of these 2 predictors are correlated. Therefore, it makes sense to fit the model with only $\log _{10}$ quoted sigma.

Fitting such a model, results in an $\mathrm{R}^{2}$ value of $30 \%$, with the coefficient on the $\log _{10}$ (quoted sigma) estimated as 1.055

This model explains over 30\% of the variation in the response, which is almost twice as much as the model with the $\log _{10}$ sample size as a response. This is still rather poor, indicating that there are other factors which explain the variation in deviations.

Though not completely satisfying all the assumptions of simple linear regression, the model performs better than the model involving sample size. The presence of outliers is apparent and these may also impact any analysis.

\subsection{CONCLUSIONS}

Quoted sigmas determine the average size of the absolute deviations better than the sample size, though sample size is a significant predictor, if quoted sigmas are not used in the model. One reason that sample size may not be such a useful predictor in regression terms is that there seems (from Figure 8.1) to be a threshold, above which, increases in sample size have little or no impact on the average absolute deviations, but below which, changes in sample size seem to have a much greater effect on the deviations. This threshold could be said to be at about $1 \mathrm{mg}$. One possible further analysis could be to look at results obtained below the sample size threshold. This may show a stronger relationship between the sample size and the deviations from the consensus. 